\title{
EVALUATING THE EFFECT OF USING MIRRORS IN 3D RECONSTRUCTION OF SMALL ARTEFACTS
}

\author{
G. Kontogianni*1, M. Lindstaedt ${ }^{2}$, T.P. Kersten ${ }^{2,}$ A. Georgopoulos ${ }^{1}$ \\ ${ }^{1}$ Laboratory of Photogrammetry, School of Rural and Surveying Engineering, National Technical University of Athens \\ 15780 Zografou Athens, Greece (gkondog, drag)@ central.ntua.gr \\ ${ }^{2}$ Laboratory of Photogrammetry \& Laser Scanning, HafenCity University Hamburg, 20457 Hamburg, Germany \\ (maren.lindstaedt, thomas.kersten)@hcu-hamburg.de
}

Commission II, WG II/8

KEY WORDS: 3D reconstruction, front surface mirror, small artefact

\begin{abstract}
:
Small artefacts pose many challenges to 3D documentation techniques due to their often complex details, which are very difficult to capture completely in 3D. Small objects may also have characteristics that are not optimal for 3D documentation, e.g. glossiness, shininess, textureless surfaces, etc. Furthermore, hidden parts of the artefact cause occlusions and obstructions, which may complicate the data acquisition process, since additional images or scan data are necessary in order to compensate for these restrictions. All these aspects increase acquisition and data processing times. Currently, the two main categories of 3D documentation methods are Image Based Modelling (IBM) and Range Based Modelling (RBM). In this paper, preliminary investigations aimed at evaluating the accuracy and performance of a front surface mirror in Image Based Modelling for small artefacts are presented. These results are then compared to a reference model generated from the artefact using a structured light system.
\end{abstract}

\section{INTRODUCTION}

Small artefacts, especially in the cultural heritage sector, present numerous details that require careful and highly accurate recording. Sometimes the procedure of $3 \mathrm{D}$ documentation is complicated due to hidden parts of the object, occlusions, and obstructions around the object. These challenges lead to increased collection and processing time, especially in cases where mass digitisation of artefacts is required.

Mirrors are often used in 3D reconstruction projects and especially in photogrammetry, where their use was firstly mentioned by Mikhail (1968). In this study, a front surface mirror was used in order to investigate the effect on the $3 \mathrm{D}$ reconstruction process for a small artefact. The object was photographed twice; once directly and then via its reflection in the mirror. Results were compared and evaluated using a 3D point cloud of a structured light system.

The state of the art for using mirrors in $3 \mathrm{D}$ reconstruction and photogrammetry is summarized in section 2, while section 3 describes the previous work of the Laboratory of Photogrammetry at NTUA in using front surface mirrors. Section 4 presents the data acquisition procedure for Image Based Modelling, including the use of a structured light system. After that, the reason for the use of the Mirror Transformation application is described in section 5, while the evaluation procedure with Multiscale Model to Model Cloud Comparison (M3C2) algorithm is presented in section 6 and Section 7 refers to some concluding remarks.

\section{STATE OF THE ART}

Mirrors are frequently used in various applications in photogrammetry and the 3D reconstruction of objects. "Mirror
Photogrammetry" was coined by Mikhail (1968), who noted that multiple perspectives can be captured without moving the camera using mirrors. The position of the camera simultaneously allows the capturing of the object and its reflection in the mirror(s). He presented four case studies in which it is assumed that the camera axis is horizontal and the object space coordinate system has the origin at the camera perspective center, its $\mathrm{X}$ axis toward the mirror and its $\mathrm{Y}$ axis along the optical axis.

In the field of orthopedics, limbs have also been modelled from photogrammetric images with the help of mirrors (Kratky, 1975). Over twenty years later, different kinds of mirrors (planar, ellipsoidal, hyperbolic and parabolic) were utilised on a four stereo system and the results compared with conventional stereo systems (Nene \& Nayar, 1998). This setup was able to acquire two or more projections of the scene in the same image, each from a single viewpoint. Furthermore, a digital close-range photogrammetry system was developed by Tokarczyk and Mikrut (2000), subsequently applied to both the medical and rail infrastructure fields. Where its application in the medical field concerned overload monitoring for medical rehabilitation, its rail infrastructure application involved locating points on crosssections. In both cases, cameras were calibrated in a laboratory. A 3D motion data analysis was developed using a system which consisted of a high-speed camera and a four-head mirror system for the determination of object coordinates (Putze, 2005). This system was employed in order to generate virtual cameras. Mitsumoto et al. (1992) used mirrors to symmetrically align the direct and mirror images in order to find correspondences between them through use of a vanishing point. They nonetheless observed that invisible parts may still remain using a mirror. However, through the use of multiple mirrors occluded parts can be made to decrease or disappear completely, thus enabling a full 3D reconstruction of an object. Moreover, Hu et al. (2005) have

\footnotetext{
* Corresponding author
} 
used planar mirrors in 3D object reconstruction. Specifically, they proved a theorem for the orientation of the mirrors and determined the distance between the mirror and the object. Riquelme et al. (2017) designed a laboratory test for analyzing the arising measurement noise when using standard and highquality mirrors in terrestrial laser scanning applications. They confirmed that the order of magnitude of the errors obtained when mirrors are used is lower than the precision of the instrument. In addition, mirrors were used in planar catadioptric vision sensors consisting of a pinhole camera observing a scene reflected in two or more mirrors. Catadioptric images were also used in SfM procedure to reliably reconstruct a large indoor scene (Doubek \& Svoboda, 2002). Mariottini et al. (2011) explored a scenario in which a robot manipulator equipped with a pinhole camera on its end-effector for observing an unknown 3D scene both directly and reflected through multiple planar mirrors. Akay \& Akgul (2014) described the problem of using multiple RGB-D cameras. They proposed the use of mirrors to introduce these cameras virtually into the system. A calibration and 3D reconstruction method based in images reflected from planar mirrors acquired with one camera was presented by Martins \& Dias (2005). A "Mirror Transformation" algorithm was developed by Thomaidis (2014) in order to generate a common point cloud by transforming the points from the plane of a front surface mirror into the point cloud. Finally, Kontogianni et al. (2018) used this "Mirror Transformation" algorithm for 3D reconstruction in two case studies, i.e. Image Based Modelling and Range Based Modelling. Schramm \& Acevedo Pardo (2010) showed that a beam geometrical observation offers solutions to some problems which can be metrologically realized by laser scanners, e.g. the surface determination of mirrors with known geometry of the reflecting objects. Their formulation of the parameterized raytracing equations allows a simple description of the imaging behavior of general mirroring surfaces for image construction and reconstruction of the originals from the images.

\section{PREVIOUS WORK}

Mirrors were used for the $3 \mathrm{D}$ reconstruction of objects in various projects at the Laboratory of Photogrammetry at NTUA. The motivation for these projects was the development of the "Mirror Transformation algorithm" (Thomaidis 2014) which allows the transformation of point clouds reflected in a mirror. Specifically, a front surface mirror was utilised for producing the reflected point cloud. This type of mirror is commonly used in industrial and scientific applications, e.g. construction of mirror stereoscopes or telescopes. These mirrors have a metallic reflective surface on the front and a glass layer on the back. They produce the best optical and metric results for all experimental procedures, since the direct interaction of the light with the reflective material means there is no refraction. However, the mirrors are sensitive since their reflective surfaces are directly exposed to the environment. Figure 1 presents a comparison of a back and front surface mirror.

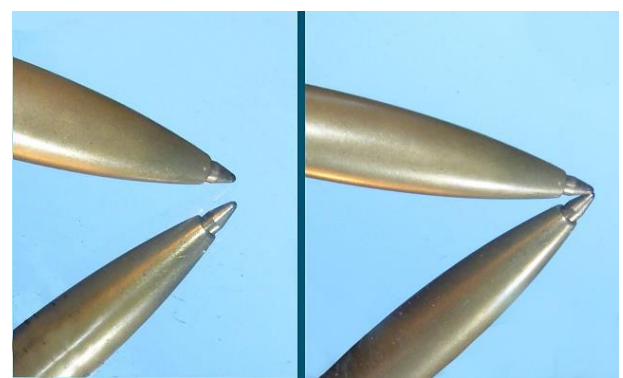

Figure 1: Effects of a back surface (left) and front surface (right) mirror) (Wikipedia, 2019)
It is obvious that in both cases the pen is touching the surface of the mirror. In the case of the back surface mirror, however, a ghosting effect is visible (Wikipedia, 2019). Thomaidis (2014) investigated in his work if and how the mirror changes the reference system of an external observer such as a laser scanner or a camera. As far as the external observer is concerned, the intervention of the mirror does not alter the orientation of the reflected artefact and as a consequence, the reference system remains the same. Thus every reflected point is connected with its corresponding original one on the object with a translation transformation. In other words, every reflected point must be moved vertically to the surface of the mirror at a distance twice its distance from the mirror surface (Thomaidis, 2014). Figure 2 illustrates this transformation.

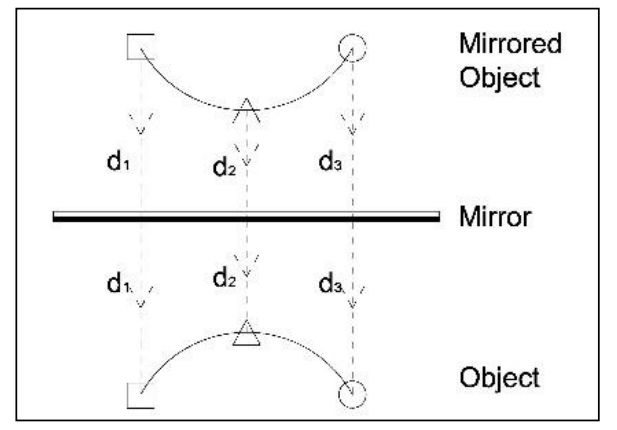

Figure 2: Translation transformation (Thomaidis, 2014)

In order to achieve the connection between the real and the reflected point cloud, the planar surface of the mirror must be precisely determined in the reference system of the external observer. The definition of the mirror's plane was carried out using four points. After the definition of the mirror's plane, the construction of the reflected point cloud was performed with the use of the translation transformation. For this purpose, the Mirror Transformation application was written in Python 2.7.6 (Python, 2013) using wxPython version 2.8 (wxPython, 2011), a Graphical User Interface (GUI) library. Additionally, the algorithm was modified to include colour information of the point cloud (Kontogianni et al. 2018).

The transformation was applied in two case studies. In the first, a Time of Flight (ToF) laser scanner was used (Figure 3).
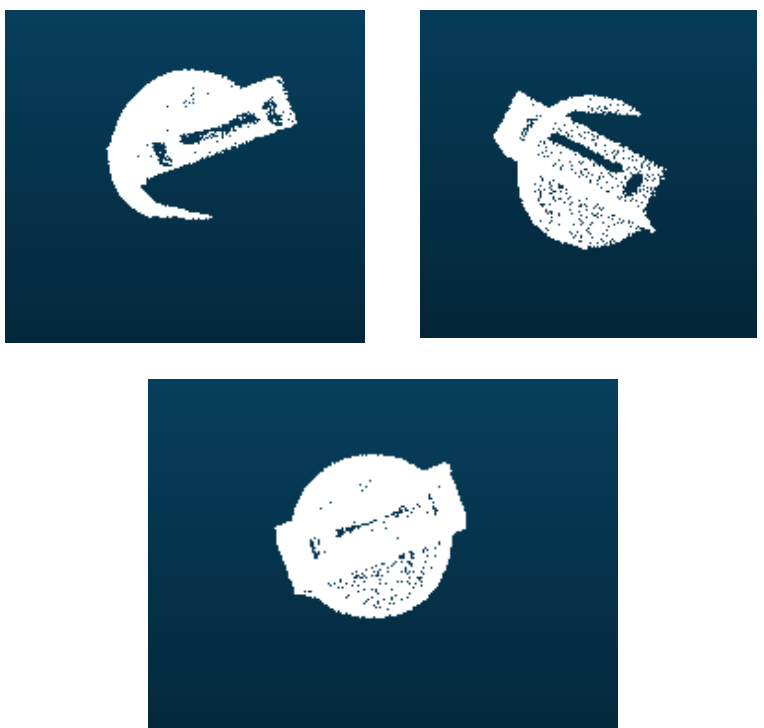

Figure 3: Point clouds before and after transformation in the case of ToF laser scanner (Kontogianni et al. 2018) 
In the second case study, Mirror Transformation was used in Image Based Modelling technique (Figure 4). In this case study, the algorithm was modified to colour information into account, as already mentioned above.
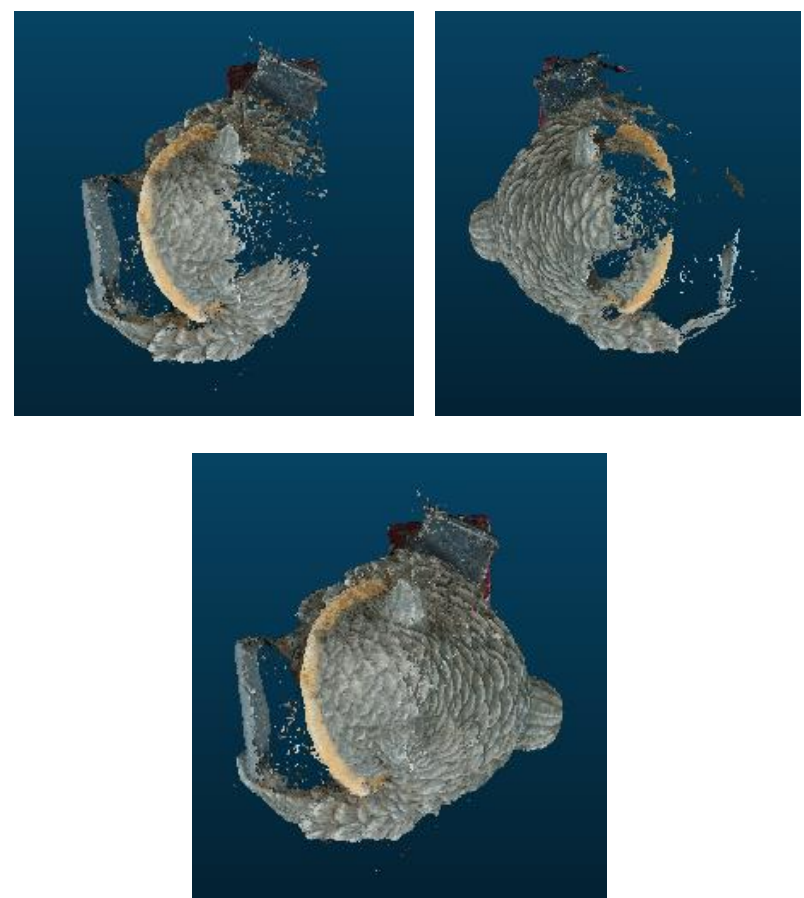

Figure 4: Point cloud before and after transformation. Image Based Modelling case study (Kontogianni et al. 2018)

\section{DATA ACQUISITION}

In order to perform the evaluation process, the object was recorded twice in 3D. For the first, the SfM-MVS algorithm was used, while in the second case study, the objects were digitised with the use of a Structured light system.

\subsection{Image Based Modelling}

In order to investigate the mirror effect on Image-Based Modelling, a front surface mirror with the dimensions $17 \times 17 \mathrm{~cm}$ was used. The mirror was placed behind the same part of the object which was photographed twice. In the first case, the part (front or behind) of the object was photographed directly and in the second case, the reflection in the mirror of the same part of the object was imaged (Figure 5).

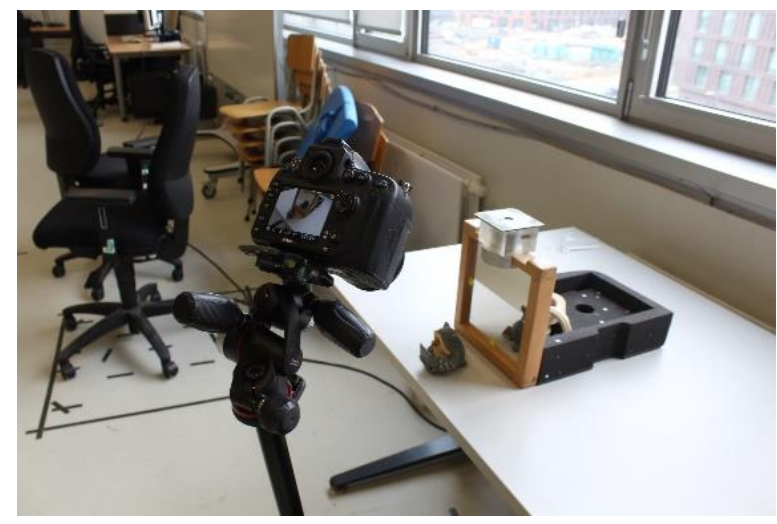

Figure 5: Image-Based Modelling experimental setup
A Nikon D800 camera with a CMOS full-frame sensor $(36 \mathrm{~mm} \times$ $24 \mathrm{~mm}$ ) and a $24-85 \mathrm{~mm}$ Nikkor zoom lens was used for data acquisition. The finely adjusted focal length was $50 \mathrm{~mm}$. The commercial software Agisoft PhotoScan (Version 1.4. 0) was employed for the creation of the 3D point cloud for each part of the artefact. Both point clouds were georeferenced in order to be projected within the same reference system. The procedure was carried out using the coordinates of the mirror's corners. Figure 6 illustrates the $3 \mathrm{D}$ point clouds for both parts, i.e. the real object and its reflection.
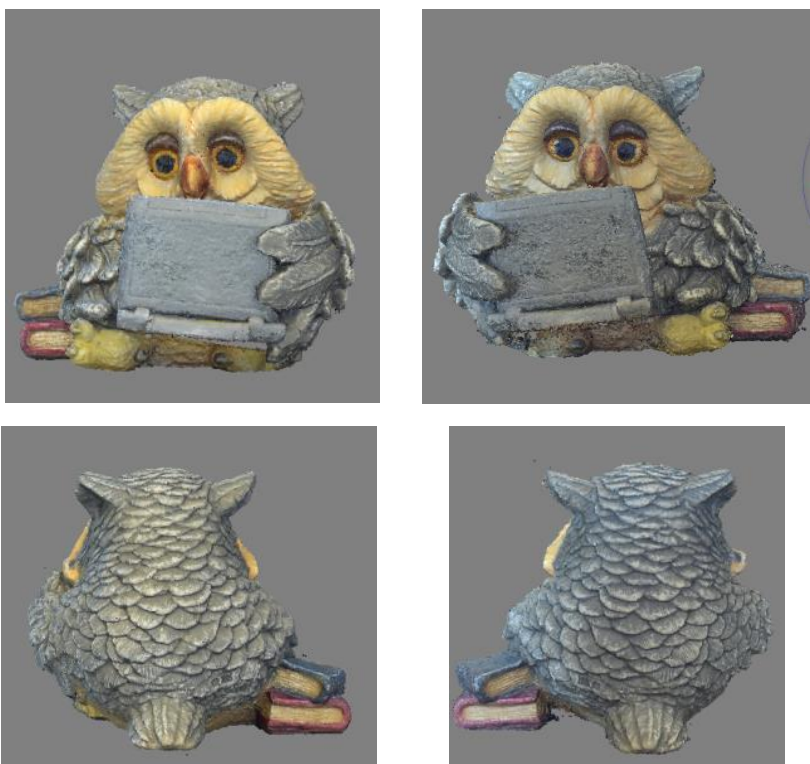

Figure 6: 3D point cloud of both parts of the real object (left) and of its reflection (right)

\subsection{Structured light system}

In order to compare the results of IBM with and without the use of the mirror, the $3 \mathrm{D}$ point cloud of the object was generated using the structured light system PrimeScan from AICON 3D Systems GmbH (Hexagon, 2019). The artefact was placed on a turntable with targets (Figure 7) in order to ensure a reliable alignment of the partial scans using the Optocat software.

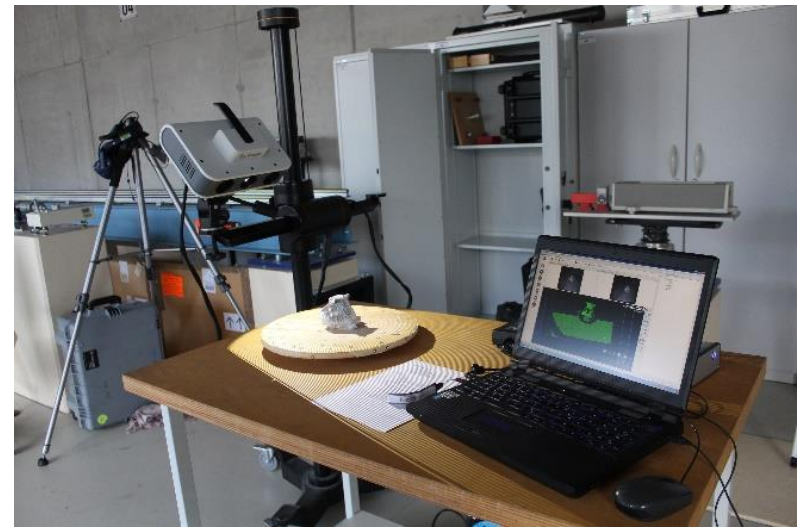

Figure 7: Structured light system experimental setup

The partial scans were then exported in ASCI format for further data post-processing in Geomagic Studio software. Two point clouds were created in total; one for the upper part and the other for the lower part of the object. The two point clouds were aligned 
and registered in order to create the 3D point cloud of the whole object. Figure 8 presents the 3D point cloud of the artefact.

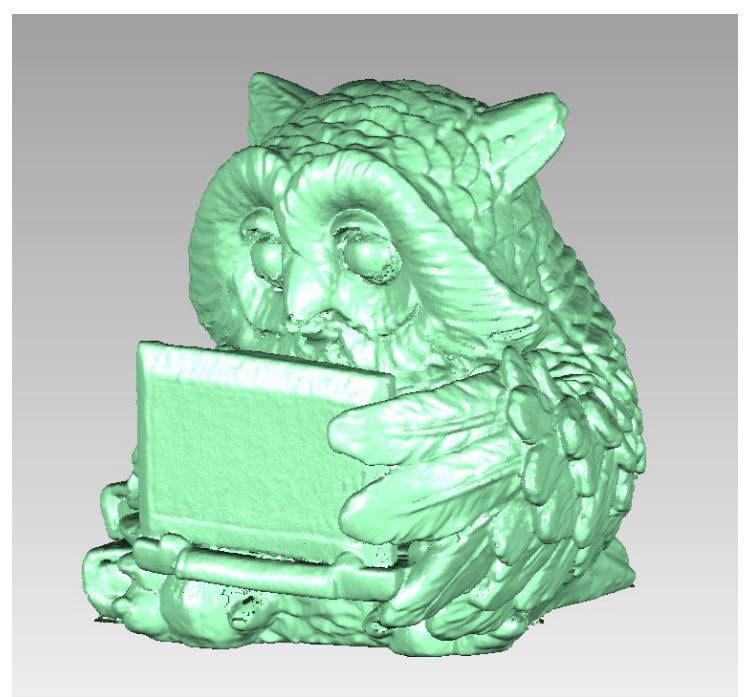

Figure 8: 3D point cloud generated with structured light system

\section{MIRROR TRANSFORMATION}

To demonstrate the effects of the mirror, the point clouds generated with IBM were registered in the same coordinate reference system as the point cloud from the structured light system (SLS) using the open source software CloudCompare (CloudCompare, 2017). Initially, the alignment process was performed by manual selection of common points in both point clouds. For the point cloud of the real object from SLS, the process was successful, though the alignment process of the reflected point cloud failed, as can be seen in Figure 9. The software recognised the reflection of the object as a different object.

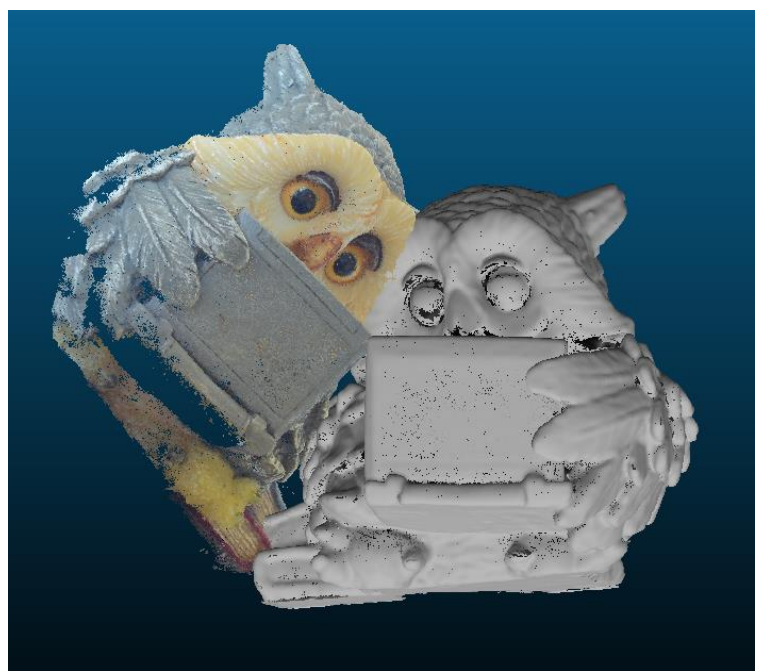

Figure 9: Alignment process between reflection and SLS point clouds

Consequently, it was investigated if the use of the Mirror Transformation application could improve the alignment process. To that end, the reflected point cloud was transformed by entering the coordinates of the four corners of the mirror, calculated with the trilateral method. The four corners of the mirror were used for the definition of its plane. After the transformation, the alignment procedure was performed to align the point clouds. Figure 10 presents the successful alignment process for the transformed point cloud of the reflection.

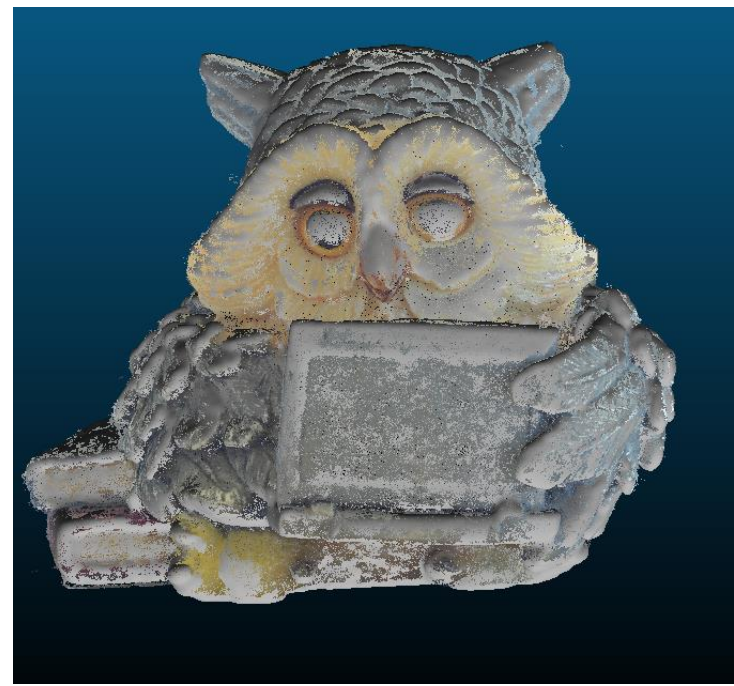

Figure 10: Successful alignment process between reflection and SL point clouds

Since the alignment is a process which connects the point clouds with minor precision, the ICP algorithm (Besl \& McKay, 1992) was used to register the two point clouds with higher accuracy.

\section{EVALUATION}

After alignment and registration stages of the point clouds, they were compared using the open source software CloudCompare. The main scope of this comparison is to investigate how the use of a high-quality mirror affects the overall process. The point cloud created with the structured light system was used as the reference data set, i.e. the 3D point clouds created with and without the use of the mirror were compared to each other. The Multiscale Model to Model Cloud Comparison (M3C2), a plugin tool of CloudCompare, was utilised to demonstrate the effect of using a mirror in the $3 \mathrm{D}$ reconstruction of small artefacts.

The M3C2 algorithm (Lague et al. 2013) operates directly on point clouds and is not dependent on their density. The algorithm uses core points in the reference point cloud. All calculations are carried out with the initial data irrespective of if the point cloud is sub-sampled or not. The computation of the distance and the confidence interval is performed for each core point.

In addition, comparisons were made between the front and back parts of the object, each photographed twice (with and without a mirror) in order to have two point clouds for each part: one for the real object and one for its reflection. Figure 11 presents the comparison that was computed between the point clouds from Image Based Modelling using the reference point cloud of the structured light system.

Surprisingly, the use of a mirror provides better results in some cases. Notably, in Figure 11 the deviations between the two point clouds are closer to zero (presented in green colour) in some regions of the back part of the object. These deviations are between $-0.5 \mathrm{~mm}$ and $+0.5 \mathrm{~mm}$. This can be seen from the colour scale, with red representing distances greater than zero and blue distances less than zero. Furthermore, in an area on the back of the artefact the mirror did not affect the final result (Figure 11). It is suspected that the structured light system did not correctly 
record the object's surface in some regions or the registration of the SLS scans was not optimally computed.
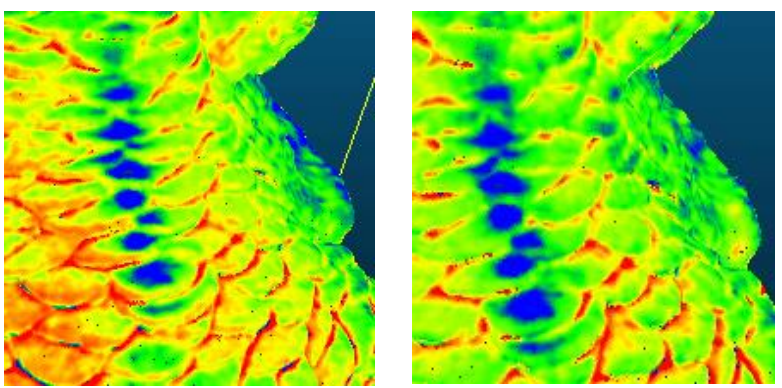

Figure 11: Region that mirror did not affect. Real object (left) and its reflection (right).

Moreover, over the front of the artefact and particularly in some areas the mirror increased the deviation between the two point clouds. For instance, the nose of the owl is redder in the reflected point cloud, as shown in Figure 12. It is possible that the reflective surface of this part was affected by the mirror. In addition, for the front of the object the use of the mirror improves the result in comparison to the direct photographing of the real object.

\section{CONCLUDING REMARKS}

In this paper, we investigated the use of a high-quality mirror for enhancing the procedure of 3D reconstruction of small artefacts. The results achieved were compared with 3D point clouds from a high-precision structured light system. It was observed that a high-quality mirror significantly affects the 3D documentation process for a small artefact, in various cases negatively or positively. The deviations obtained allow the use of high-quality mirrors in $3 \mathrm{D}$ documentation procedures for small artefacts. It is
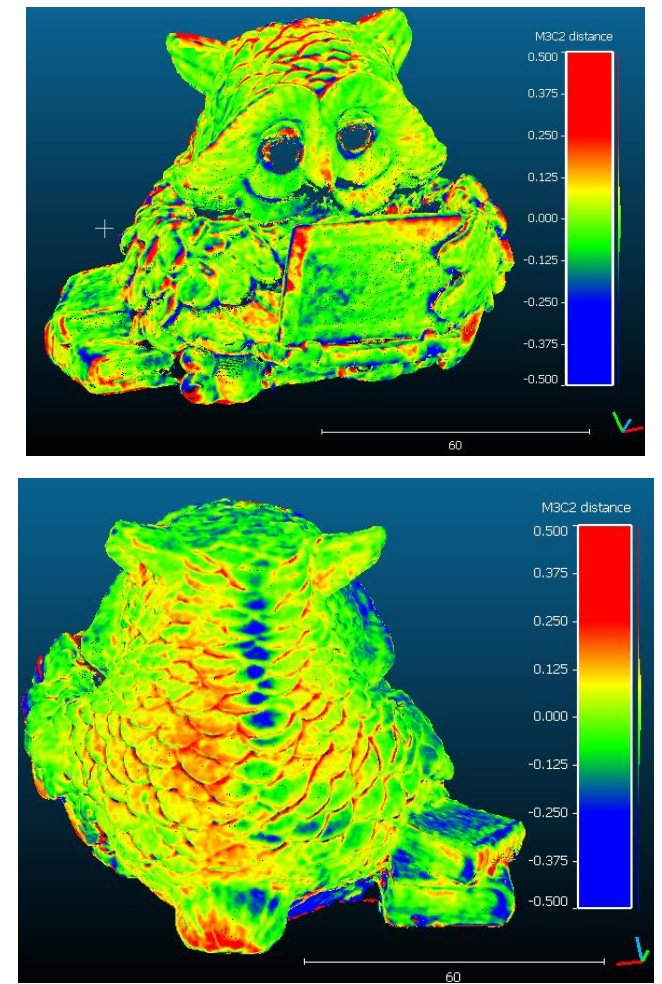

possible that these differences are due to surface characteristics, e.g. the glossiness of the object or the lighting of the scene.

Hence, a proper experimental setup should be considered to achieve reliable and precise results. Furthermore, mirrors are a very useful tool if areas of an object are difficult to photograph or if occlusions and obstructions complicate the image acquisition procedure. Moreover, mirrors can be utilised in efficient mass digitisation procedures.

Finally, the use of additional mirrors positioned around the object will speed up the process of image data acquisition and 3D reconstruction. Furthermore, it is suggested to automate the procedure of point cloud registration and Mirror Transformation using coded targets for automatic detection and decoding. This aspect will significantly support the use of multiple mirrors for image data acquisition.

\section{ACKNOWLEDGEMENTS}

The research has been financially supported by the General Secretariat for Research and Technology (GSRT) and the Hellenic Foundation for Research and Innovation (HFRI).

Also, the authors would like to thank Alexander Walmsley for checking and editing the English spelling of this paper.

Figure 12: Comparisons with the $\mathrm{M} 3 \mathrm{C} 2$ algorithm real part of the object (left) and the reflection (right) 


\section{REFERENCES}

Akay, A., Akgul, Y.S., 2014. 3D reconstruction with mirrors and RGB-D cameras. In IEEE International Conference on Computer Vision Theory and Applications (VISAPP), Lisbon Portugal, Vol. 3, pp. 325-334.

Besl, P.J., McKay, N.D., 1992, Method for registration of 3-D shapes. In Sensor Fusion IV: Control Paradigms and Data Structures, Vol. 1611, pp. 586-607.

CloudCompare, 2017. 3D point cloud and mesh processing software. https://www.danielgm.net/cc/ (6/6/2019)

Doubek, P., Svoboda, T., 2002. Reliable 3d reconstruction from a few catadioptric images. Proceedings of the IEEE Workshop on Omnidirectional Vision. Copenhagen Denmark, pp. 71-78.

Hu, B., Brown, C., Nelson, R., 2005. Multiple-view 3-D reconstruction using a mirror. Technical Report TR863, Computer Science Dept. University of Rochester

Kontogianni, G., Thomaidis, A. T., Chliverou, R., Georgopoulos, A., 2018. Exploiting Mirrors in 3D Reconstruction of Small Artefacts. The International Archives of the Photogrammetry, Remote Sensing and Spatial Information Sciences, Vol. 42(2), pp. 531-537, https://doi.org/10.5194/isprs-archives-XLII-2-5312018.

Kratky, V., 1975. Digital modelling of limbs in orthopedics. In: Photogrammetric Engineering \& Remote Sensing, Vol. 41, pp. 741-752.

Lague, D., Brodu, N., Leroux, J., 2013. Accurate 3D comparison of complex topography with terrestrial laser scanner: Application to the Rangitikei canyon (NZ). ISPRS journal of photogrammetry and remote sensing, Vol. 82, pp.10-26.

Mariottini, G.L., Scheggi, S., Morbidi, F., Prattichizzo, D., 2012. Planar mirrors for image-based robot localization and 3-D reconstruction. Mechatronics. Elsevier, Amsterdam, Vol. 22(4), pp. 398-409.

Martins, N., Dias, J., 2004. Camera calibration using reflections in planar mirrors and object reconstruction using volume carving method. The Imaging Science Journal, Vol. 52(2), pp. 117-130. DOI: $10.1179 / 136821904225011609$

Mikhail, E.M., 1968. Principles and Applications of Analytical Mirror Photogrammetry. Journal of the American Society of Photogrammetry, Vol. 24(10), pp.1071-1078.

Mitsumoto, H., Tamura, S., Okazaki, K., Kajimi, N., Fukui, Y., 1992. 3-D reconstruction using mirror images based on a plane symmetry recovering method. IEEE Transactions on Pattern Analysis and Machine Intelligence, Vol. 14(9), pp. 941-946. DOI: $10.1109 / 34.161352$

Nene, S.A. and Nayar, S.K., 1998. Stereo with mirrors. In Sixth IEEE International Conference on Computer Vision, Bombay India, pp.1087-1094. DOI: 10.1109/ICCV.1998.710852

Putze, T., 2005. Geometric modelling and calibration of a virtual four-headed high speed camera-mirror system for 3-D motion analysis applications. Optical 3-D measurement techniques VII, Vienna Austria, pp. 167-174.

\author{
Python, 2013. Python Release \\ https://www.python.org/downloads/release/python-276/ \\ $(6 / 6 / 2019)$
}

Riquelme, A.J., Ferrer, B., Mas, D., 2017. Use of High-Quality and Common Commercial Mirrors for Scanning Close-Range Surfaces Using 3D Laser Scanners: A Laboratory Experiment. Remote Sensing,Vol. 9(11), pp.1152-1165.

Schramm, T., Acevedo Pardo, C., 2010. Reflexionen über Spiegel. Photogrammetrie, Laserscanning, Optische 3DMesstechnik, Beiträge der Oldenburger 3D-Tage 2010, Th. Luhmann, T. \& Müller, Ch. (Eds.), Verlag Herbert Wichmann, pp. 309-316 (In German).

Tokarczyk, R., Mikrut, S., 2000. Close range photogrammetry system for medicine and railways. The International Archives of Photogrammetry and Remote Sensing and Spatial Information Sciences, Vol. 33(5), pp. 519-524.

Thomaidis, A.T., 2014. Scanning with Mirrors. Diploma Thesis, National Technical University of Athens, Athens Greece. (In Greek)

White Light Scanner Systems- AICON PrimeScan, 2019 https://www.hexagonmi.com/products/white-light-scannersystems/aicon-primescan (5/6/2019) Wikipedia, $2019 . \quad$ First
https://en.wikipedia.org/wiki/First_surface_mirror $(15 / 6 / 2019)$

WxPython, 2011. WxPython library version 2.8 . https://wxpython.org/ (10/5/2019) 